\title{
How to drive passenger airport experience: a decision support system based on user profile
}

ISSN 1751-956X

Received on 13th July 2017 Revised 7th November 2017 Accepted on 5th January 2018 E-First on 12th February 2018 doi: 10.1049/iet-its.2017.0210 www.ietdl.org

\author{
Riccardo Rossi ${ }^{1} \bowtie$, Massimiliano Gastaldi ${ }^{1}$, Federico Orsini ${ }^{1}$ \\ ${ }^{1}$ Dipartimento di Ingegneria Civile Edile e Ambientale, Università degli Studi di Padova, Via Marzolo 9, 35131 Padova, Italy \\ 凶-mail: riccardo.rossi@unipd.it
}

\begin{abstract}
This work presents a decision support system for providing information and suggestions to airport users. The aim of the study is to design a system both to improve passengers' experience by reducing time-spent queuing and waiting and to raise airport revenues by increasing the time passengers spend in discretionary activities. Passengers' behaviour is modelled with an activity-choice model to be calibrated with their mobile phone traces. The model allows predicting activity sequences for passengers with given socio-demographic characteristics. To predict queue length at check-in desks and security control and congestion inside commercial areas, passengers' movements are simulated with a microscopic simulation tool. A system to generate suggestion has been designed: passengers are advised to perform mandatory activities when the predicted queue length is reasonable and specific discretionary activities according to time available, user profiles, location distance, location congestion and airport management preferences. A proof-of-concept case study has been developed: passengers' behaviour in both cases of receiving and not receiving suggestion has been simulated. In the first case, passengers experienced less queuing and waiting time; the time saved was spent in discretionary activities, improving passengers' airport experience and increasing airport revenues.
\end{abstract}

\section{Introduction}

According to the European Organisation for the Safety of Air Navigation [1], the number of flights in Europe will increase, from 9 million in 2012 to 14.4 million by 2035. The European Commission itself promotes the use of existing infrastructure to improve airport capacity, as efficiency has a great impact on both customer satisfaction and the financial performance of airports [2]. In addition, airports are one of the main indirect sources of income for a country, since they generate employment, attract investment, stimulate tourism and contribute to world trade [3]; in Europe, airports support $4.1 \%$ of the gross domestic product [4]

An airport is a complex environment in which pedestrians move according to a schedule that contains a number of mandatory activities (check-in, security control, etc.) and, depending on available time, also a number of discretionary activities (eating, shopping, etc.). Airport passengers may experience feelings of anxiety, stress, and excitement, which make them react in unusual ways so that they do not behave like ordinary shoppers in a typical commercial area situation [5].

It is reported that a pleasant airport experience encourages spending and influences future travel plans [6]. Retail concession business is often an important element in the overall financial portfolio of companies operating in airports [7]. In some cases, non-aviation revenues account for as much as $53 \%$ of total revenues [5]. It is therefore important for an airport manager to improve passengers' experience, as this can also have a positive outcome on revenues.

This work presents a decision support system for providing information and suggestions to airport users. The aim of the study is to design a system, which can both improve passengers' experience, by reducing time spent queuing and waiting, and raise airport revenues, by increasing the time passengers spend in discretionary activities.

Passengers' behaviour is modelled with an activity-choice model; its calibration has been thought as based on passengers' mobile phone traces. The model allows analysts to predict activity sequences for passengers with given socio-demographic characteristics. To predict queue length at check-in desks and security control and congestion inside commercial areas, passengers' movements are simulated with a microscopic simulation tool.

A system to generate suggestion has been designed and developed: passengers are advised to perform mandatory activities when the predicted queue length is reasonable, and specific discretionary activities depending on time available, their tastes, location distance, location congestion and airport management preferences.

The paper is organised as follows. Section 2 briefly describes the theoretical background; Section 3 presents the general framework, divided into two main parts: the activity choice and the suggestion generation; Section 4 contains a proof-of-concept casestudy illustrating the whole framework. Section 5 concludes with some remarks and predicted future developments.

\section{Background}

To date, a number of models have been developed to simulate passengers' behaviour inside airport terminals, and are classified as macroscopic or microscopic. The former is often used to analyse queues [8]; the latter is necessary for reliable and detailed evaluation of the complex movements of every passenger [9].

Recently, some projects focusing on airport passengers have been carried out: Airport of the Future Project [10] focuses on safety, security, and efficiency, and has been applied in Australian airports; Proactive Passenger Flow Management for Airports with an Advanced Forecasting System [11] has developed a tool for airport management, by predicting passenger flows inside the terminal; and DORA [12] is an extended project to reduce passengers' travel time, providing them with real-time route information from the origin (e.g. their home) to the aircraft.

Starting from literature considerations and the assumptions briefly described above, this research project aims at integrating simulation of passenger flows and information on route choices provided to passengers inside airport terminals. To model pedestrian demand, which is driven by the need to perform activities in various locations, this work focuses on applying activity-based models to airport passengers.

Although much pedestrian research has been published, it mainly focuses on walking behaviour and crowd dynamics, not on 


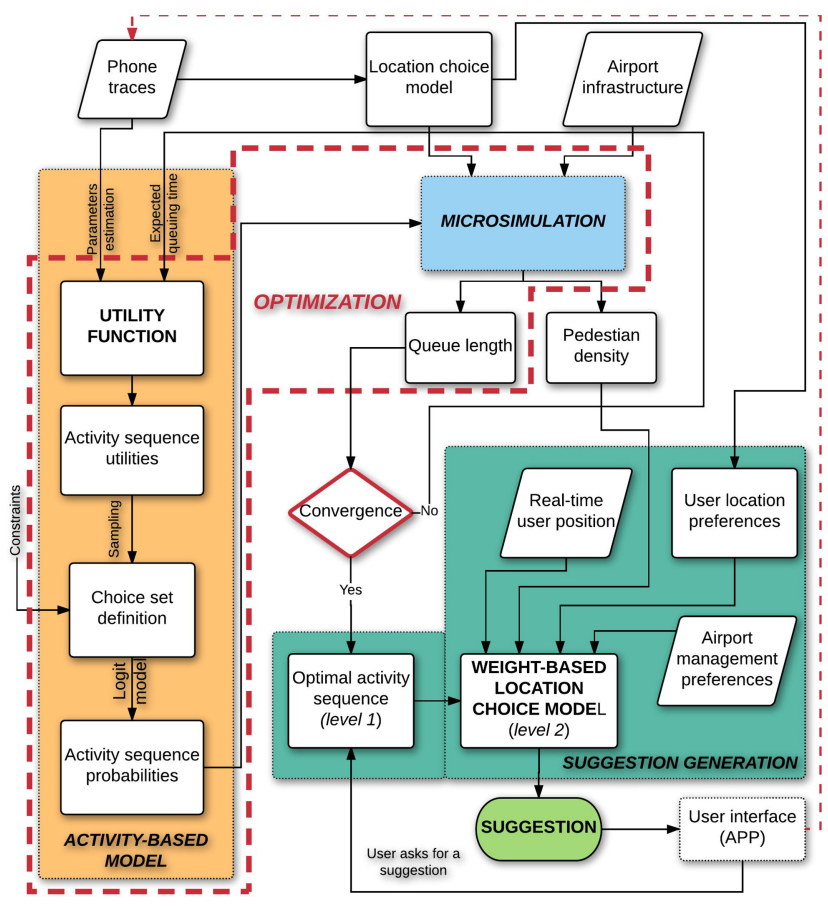

Fig. 1 Overall structure of the framework

activity choice. Bierlaire and Robin [13] present a general review of pedestrian research, dividing walkers' behaviour into six steps: activity, destination, mode, route, next step and speed choices. From their review, it seems that the least studied step is activity choice.

In another significant paper on the subject, Hoogendoorn and Bovy [14] define three pedestrian behavioural levels:

- Strategic, which includes departure time and activity pattern choices.

- Tactical, involving activity scheduling, activity area choice, and route-choice to reach activity areas.

- Operational, i.e. walking behaviour.

In this hierarchy, expected utilities at lower levels influence choices at higher levels, while choices at higher levels affect choice sets at lower levels.

Their paper focuses on the tactical level, as the operational level had already been covered by Hoogendoorn [15]. However, the strategic level is not examined and pedestrian arrival patterns, distinguishing groups of pedestrians having similar characteristics (activity sets, travel purpose, demographic characteristics, etc.) are assumed to be known a priori.

An interesting 'strategic level' approach was recently developed by Liu et al. [16], who presented an activity-based travel demand model within the context of an airport, focusing on activity scheduling, destination and route choices and rescheduling models, based on revealed and stated preference survey data. The typical home-based structure of car-centric activity-based models is here replaced by a nested structure which divides possible activities at the airport into three phases: before check-in, before security, and before boarding. They examine the behaviour of passengers with differing socio-demographic characteristics (e.g. gender, age, education, etc.) and flight characteristics (low-cost/ordinary airline, number of pieces of luggage, etc.). Possible discretionary activities at the airport are divided into five types. In this model, the output is the probability that a given activity type is performed by a given passenger type in a given time phase. The model cannot predict activity duration or take into account time-of-the-day preferences.

Kalakou and Moura [17] focused on passengers' activity choice before security control. A multinomial logit model was estimated to explain passengers' choices regarding activities in the terminal and, more specifically, whether they choose to perform only aeronautical activities before passing the security checkpoint or both aeronautical and non-aeronautical activities. Data were collected with a revealed preference survey. They did not model activity duration.

In the micro-simulation model of Ma [18] passengers are constructed as intelligent agents who possess some initial basic traits and can, therefore, be categorised into ten groups in terms of route-choice preferences, by inferring the results of advanced traits. These traits represent passengers' cognitive preferences, i.e. level of hunger, relaxation, willingness to ask for help, etc. The study used empirical data to validate the model, the use of surveys and video cameras was suggested.

In his general review on activity-based models, Danalet [19] stressed that most of them are not directly applicable to model pedestrian behaviour because they have a 'home' location and a tour-based structure: this is the case of many models (e.g. [20-26]).

In addition, the choices of activity types and schedules are often not considered simultaneously, for combinatorial reasons [27]: when examining simultaneously activity type and scheduling, assuming that the period of interest contains $\mathrm{T}$ time units, the number of alternatives is $K^{\mathrm{T}}$ and the choice set size increases exponentially. Considering tours or sequential order, without timing, the size of the choice set decreases.

Danalet [19] developed a new model to fill this gap in the past literature. Its main strengths are as follows:

- The absence of a home and tours-from-home structure.

- The absence of priority between activities (e.g. primary vs secondary).

- Management of large choice sets, by important sampling (Metropolis-Hastings algorithm [28]). This allows to simultaneously modelling the choice of activity type, time-ofthe-day, and duration.

- The use of a single utility function for activity type, time-of-theday preferences, and duration. Represented as a path in a network, the sequence is a single choice and utility is associated with the full pattern.

For the above mentioned reasons, Danalet's model [19] was used as a starting point for the present work. The activity types and passenger characteristics of Liu et al. [16] influenced the definition of the specific airport case-study scenario.

\section{Model framework}

Fig. 1 represents the overall framework of the proposed model. It is divided into two main parts:

- activity choice and micro-simulation models predict passenger behaviour and movements inside the airport terminal; and

- a suggestion generation model gives passengers personalised information, depending on user profile, queue length prediction, user position, and airport management interest.

The system is thought to be applied, at least initially, to a class of profiled users composed by a limited number of subjects, i.e. the frequent-flyer-card users of an Italian airport which has expressed interest in adopting this system. These users, changing their behaviour after receiving suggestions, do not significantly modify queue length prediction.

However, in view of a future extension to a wider user base, an optimisation process is included in the system (red-dashed polygon in Fig. 1). The objective is to maximise the activity sequence utility for each passenger, with one of the constraints being the suggestions given to the other passengers: the idea is to reach an equilibrium condition, through an iterative process of utility adjustment, where no individual passenger can increase her/his own utility by switching sequence of activities.

The following sections present a detailed description of the system components, with focus on those applied in the case-study (see Section 4). 

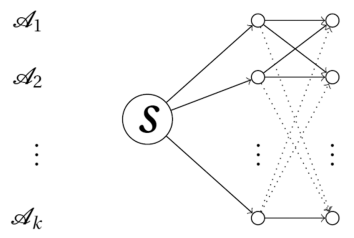

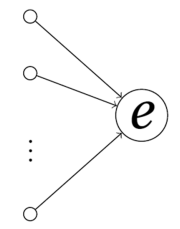

$\begin{array}{lllll}1 & 2 & \cdots & T & \text { Time units }\end{array}$
Fig. 2 Activity network. Each node is an activity of type $k$ performed at time $t$ [19]

\subsection{Activity choice model}

In the activity choice model, all possible activity patterns are represented in a network. The model was developed by Danalet [19]; this work makes a further contribution by adapting it to the case of an airport.

The network is shown in Fig. 2. It is discrete with respect to activity types and time.

All activities, which can be performed at the pedestrian facility, are categorised as various activity types $A_{1}, A_{2}, \ldots, A_{K}$, for a total of $K$ types. For example, in an airport scenario, these activities can be: performing a mandatory activity (check-in, security control), eating, shopping, waiting, using the toilet, etc. Later in this section, the specific activity types of this paper's case-study will be described in detail.

After being defined, the time horizon (which, according to the type of application, may be some minutes, some hours, an entire day or even more) is divided into $T$ time units. All time units usually have the same duration, although not necessarily [29]. Time unit duration is closely related to the specific case-study.

There are two special nodes in the network, $s$ and $e$. Node $s$ represents the starting node for every path in the network; it is located before time unit 1 and does not belong to any single activity type, but is linked to all activity types available at time 1 . Node $e$ is the end node for every path, is located after time unit $T$ and is linked to all activity types available at time $T$.

The generic node $A_{k t}$ of the network represents activity $k$ performed by an individual at the time unit $t$. Links connect nodes and represent activities $A_{k t}$ performed in order. Each node at time unit $t$ is linked to every node at the following time unit $t+1$; this represents the choice of changing activity type or maintaining it for one more time unit.

An activity episode $a=\left(A_{k}, t^{-}, t^{+}\right)$in the activity network is defined as an activity type $A_{k}$ which the user is performing from start time $t^{-}$to end time $t^{+}$. The length of an activity episode $a$ is $a=t^{+}-t^{-}+1$.

Activity paths $A_{1: T}$ represent activity patterns in the network and are the alternatives in the choice process. Each activity path contains $T$ nodes (plus $s$ and $e$ ) and $N_{a}$ activity episodes, which are less than or equal to the number of nodes in the path.

In total the network contains $K T+2$ nodes, $2 K+K^{2}(T-1)$ links and $K^{T}$ paths. A logit model is adopted to obtain the probability of being chosen for each path, according to a utility function. Clearly, the universal choice set cannot be used because of its size, so that some kind of sampling strategy must be used to reduce the number of alternatives.

Choosing between activity path alternatives allows the analyst to model the activity generation and activity schedule at the same time.

The deterministic part of utility is divided into three components, as typically found in the literature (see [19, 30]): the time-of-the-day preference, satiation effect and repetition effect. The general equation for the utility is

$$
V_{\Gamma}=\sum_{k=1}^{K} \sum_{t=1}^{T} \sum_{g=1}^{G} V(A)+\sum_{a=1}^{N_{a}} \sum_{g=1}^{G} V(a)+\sum_{g=1}^{G} V(\Gamma) .
$$

The utility obtained with this equation is specific for path (activity sequence) $\Gamma$ and pedestrian group $g$.

The first element in the utility is time-of-the-day preference and indicates the individual marginal utility of allocating a time unit to a certain activity type. The time-of-the-day utility depends not only on activity type $k$ but also on time $t$ at which the activity is performed. It is a sum of $K^{*} T^{*} G$ components

$$
V(A)=\beta_{k t g} I_{k t} I_{g}
$$

where $\beta_{k t g}$ is the generic parameter (to be calibrated), which changes as a function of pedestrian group, activity type and over time. $I_{k t}$ is a dummy variable having value 1 when activity $A_{k t}$ belongs to path $p$ whose utility is being computed, and 0 otherwise. $I_{g}$ is another dummy variable having value 1 when the utility refers to pedestrian group $g$ and 0 otherwise.

The second element is the satiation effect, which takes into account the 'fatigue' of performing an activity. The satiation effect depends on the type and length of the activity episode and the pedestrian group. Its equation is as follows:

$$
V(a)=\eta_{k g} \ln |a| I_{k} I_{g}
$$

where $\eta_{k g}$ is the set of parameters to be calibrated: they change for every activity type and pedestrian group and generally have a negative value to represent disutility. The first dummy variable $I_{k}$ is 1 when episode $a$ belongs to activity type $k$, and 0 otherwise. The second dummy variable $I_{g}$ is 1 when the pedestrian in question belongs to group $g$, and 0 otherwise. The term is then multiplied by the natural logarithm of the activity episode length.

The third element is the repetition effect and accounts for the effect of repetition of the same activity in the activity sequence. $|k|$ is the number of repetitions of a given activity type $k$

$$
V(\Gamma)=\beta_{|k| g} I_{|k|} I_{g},
$$

where $\beta_{|k| g}$ is the calibrated parameter, depending on passenger group $g$ and the number of repetitions of activity $k$. There are also two dummy variables, the first is 1 when the sequence contains an activity $k$ repeated $|k|$ times, and 0 otherwise. The second is the same as in time-of-the-day and satiation effects and is 1 when the individual belongs to pedestrian group $g$, and 0 otherwise.

The model is hypothesised to be calibrated according to pedestrians' mobile phone traces. These traces must be preprocessed; several procedures are reported in the literature [31, 32].

In the model estimation, the whole choice set cannot usually be used, due to its size. Danalet [19] proposed using important sampling, specifically the Metropolis-Hastings algorithm [33], adapted to path sampling by Flötteröd and Bierlaire [28]. The Metropolis-Hastings algorithm generates a Markov chain with a predefined stationary distribution. Crucially this distribution need not be normalised and therefore all the paths in the network need not be enumerated.

The algorithm intuitively works as follows: given an origin and a destination in the network, an arbitrary (e.g. the shortest) path between the nodes is first assumed; the algorithm then repeatedly carries out random modifications of the path; according to the known probability of each random modification, this modification is either accepted or rejected. This decision is made so that the long-term frequency of the occurrence of each path in the process coincides with the analyst's specification. For all details about the Metropolis-Hastings sampling of paths see [28].

Path utility values are used to compute the probability of any given path $\Gamma$ being chosen by a passenger belonging to group $g$. A logit model is used 


$$
P\left(\Gamma_{g}\right)=\frac{\mathrm{e}^{V_{\Gamma}}}{\sum_{p=1}^{N_{\Gamma}} \mathrm{e}^{V_{p}}},
$$

The number of paths $N_{\Gamma}$ appearing in the equation is the total number of sampled paths.

These activity choice probabilities can then be applied in a micro-simulation software to represent the movement of pedestrians and to obtain a number of outputs, e.g. queuing times or pedestrian densities in selected areas.

In this airport case study, six different activity types are defined, similar to those defined by Liu et al. [16]

- Mandatory: walking towards an active service point (e.g. security checkpoint, boarding gate when it is open), standing in a queue, being serviced.

- Eating: all activities involving moving to a point where food and drink are provided in some form (restaurants, fast food outlets, bars, automatic machines, etc.).

- Shopping: any activity during which passengers move to and visit sales outlets.

- Other: all other activities performed at the airport not included above, e.g. using the toilet facilities, asking for information.

- Waiting: moving to and staying in a waiting area, e.g. in the entrance hall or in front of the gate. This includes queuing in front of the gate before boarding procedures begin.

- Not-at-the-airport: passengers are not inside the airport's pedestrian areas, i.e. they are outside the system.

For all passengers, the time horizon is defined as a span of time before the scheduled flight departs. So end time is set as the time at which the flight should depart; start time is set some hours earlier, in order to ensure that a high percentage of passengers are included in the model (i.e. they arrive at the airport after the start time). To set the time horizon precisely, analysts should observe available data, particularly passenger arrival times, set a percentage of passengers to be included (e.g. 95 or 99\%) and define the start time accordingly.

Time unit length should also be chosen by examining the observed sequences and it should be small enough not to 'miss' episodes: if an activity episode starts and finishes between successive time units, it cannot be modelled. To avoid missing any activity, very high time resolution (i.e. low time unit length) could theoretically be chosen but, if the value of $T$ is large, the network dimension 'explodes', as the number of paths in the network is $K^{T}$. It is therefore important to keep this number as small as possible, without losing too much precision for the activity types of interest.

In an airport passengers can be grouped according to several characteristics, e.g. gender, age, flying frequency, travelling in a group or not, arriving late or early, with online check-in or not, using a low-cost or a traditional airline, etc. A combination of these characteristics can identify several types of passenger groups. The number of groups $G$ greatly influences the number of parameters to be estimated, because the time-of-the-day, satiation and repetition parameters of each group must be estimated. This also influences the size of the dataset needed for estimation, pre-processing and calibration times.

\subsection{Suggestion generation}

The second component of the framework is an 'engine' which generates a personalised suggestion for pedestrians, based on their user profiles (i.e. pedestrian groups) and the outputs obtained from the activity choice model and the micro-simulation. The method presented here targets airport passengers, but can also be adapted to other scenarios. The model for suggestion generation is divided into two levels: (1) activity sequence suggestion; (2) location choice suggestion.

The first and more important part of the model is to generate the new activity sequence, which passengers should follow to reduce their queuing and waiting times.

For each passenger group, the activity choice model of Section 3.1 indicates which activity sequence has the highest utility.
However, this sequence is not necessarily the 'best', but is the most frequently chosen sequence, since the utility function is calibrated with observed data. The target is to improve this sequence and thus minimise queuing and waiting times. First, however, it is necessary to modify some variables in the utility function, and then find the new 'best' sequence.

While most of the activity types in the utility function have set time-of-the-day parameters, the values of which are $\beta_{k t g} I_{k t} I_{g}$, 'mandatory' and 'waiting' activity types have a different formulation.

The mandatory activity time-of-the-day parameters are

$$
V(A)_{1}=\left(\beta_{1 t g} I_{1 t}+\gamma_{1 g} \mathrm{EQT}_{t}\right) I_{g},
$$

where $k=1$ means that only mandatory activity parameters are considered, EQT is the variable 'expected queuing time' and $\gamma_{g}$ is an estimated parameter.

With this EQT term, it is assumed that passengers have their own 'prediction' on how the queuing times are going to evolve and they are thus influenced by them when deciding exactly when to pass through security control. As the micro-simulation tool output can predict how queue length will actually evolve, the value of this set of variables is modified, by replacing the 'expected times' with the 'predicted times'. All the parameters, including $\gamma$, remain unchanged. The effect is that the new variables' values modify the utility value of all the paths in the activity network, assigning higher values to activity sequences in which the mandatory activity is performed when the queue length is low, and vice versa.

The 'waiting' time-of-the-day terms can also be modified in a similar way. Their formulation becomes

$$
V(A)_{5}=\left(\beta_{5 t g} I_{1 t}+\gamma_{5 g} I_{\text {info }}\right) I_{g}
$$

where $k=5$ means waiting activity. $I_{\text {info }}$ is a dummy variable equal to 1 when passengers receive information regarding available time, discretionary activities, etc. and 0 if no information is given. In this way, the overall utility of sequences with long waiting times in front of the gate is reduced.

After these variables have been modified, new utility values are obtained for the paths in the network. At this point, it is necessary to find the best activity sequence for each passenger group. If the utility function is correctly calibrated, a new activity sequence is expected to appear, in which waiting and queuing times are reduced, with respect to the previous best activity sequence. Since it is only necessary to identify the best path in the activity network, a shortest-path algorithm can be applied. The problem is that only a portion of the elements in the utility function is link-additive: satiation effect and repetition effect parameters are not. This prevents the analyst from applying a traditional shortest-path algorithm; however, non-additive shortest path algorithms are reported in the literature (see $[34,35]$ ). Alternatively, the highest utility path can be chosen from a limited set of possible paths, obtained with a rule-based method. Further tests will identify the most effective approach.

Assuming that the best activity sequence, which passengers must follow, is known, it is then necessary to identify the best locations in which to perform the activity episodes in the sequence.

A weight-based location choice model is developed, in which a total weight $W$ is assigned to each location $i . W$ is a function of other weights $\mathrm{BW}, \mathrm{CW}, \mathrm{UW}$ and $\mathrm{AW}$ and changes with time $t$

$$
W_{i t}=f\left(\mathrm{BW}_{i t}, \mathrm{CW}_{i t}, \mathrm{UW}_{i}, \mathrm{AW}_{i}\right),
$$

where

- BW is the base weight, and depends on passengers' real-time position compared with location $i$ and time availability.

- $\mathrm{CW}$ is the congestion weight, and depends on the number of people performing an activity at location $i$.

- $\mathrm{UW}$ is the user weight, and depends on the tastes of passengers belonging to a given passenger group. 
- AW is the airport weight, and depends on which locations the airport management wishes to advertise.

The user, at time $t$, will receive the suggestion to visit the location with the highest total weight $W$. Alternatively, a list of possible locations, sorted according to the total weight, may be provided to the user.

By default, the system will decide the time $t$ when to give suggestions, but the user will have the ability to 'ask' the system for a suggestion at any time $t$.

\subsubsection{Base weight: The proposed equation for $\mathrm{BW}$ is}

$$
\mathrm{BW}_{i t}= \begin{cases}-\frac{\mathrm{BW}_{\max }}{T_{t}-t_{p i}} D_{i}+\mathrm{BW}_{\max }, & \text { if } T_{t}-t_{p i}>0, \\ 0, & \text { if } T_{t}-t_{p i} \leq 0 .\end{cases}
$$

Given a user's position at time $t$, BW for location $i$ decreases with distance $D_{i}$ between user and location, according to time availability $T_{t}$, which depends on the allocated remaining time in the 'best' activity sequence.

All locations are also associated with a value $t_{p i}$ which represents the minimum time required to perform an activity at location $i$. That is, if there is not enough time to perform an activity at location $i$, the user never receives a suggestion to go there.

$\mathrm{BW}_{\max }$ is a parameter, which must be set by the analyst. It 'weights' the importance of BW in the calculation of overall $W$.

In the equation, distance is expressed in walking time. $D_{0}$ represents the limit distance at which $\mathrm{BW}=0$. Locations farther than $D_{0}$ will never be suggested, because there is not enough time to reach them, in view of time availability $T_{t}$ and minimum time $t_{p i}$.

3.2.2 Congestion weight: Another aspect, which must be considered when making suggestions to users, is the degree of saturation inside possible destinations. Priority is given to less crowded places: this has a positive effect on both users and airport management, e.g. passengers will spend less time queuing for coffee or waiting for their meal to be prepared.

In the equation proposed here, $\mathrm{CW}$ decreases linearly with congestion inside location $i$ at time $t+\tau$

$$
\mathrm{CW}_{i t}=c\left(-\frac{n_{i t+\tau}}{N_{i}}+1\right)
$$

where $\mathrm{CW}$ is computed at time $t$ when the passenger receives the suggestion. It takes into account capacity $N_{i}$ of location $i$ and the number of passengers $n_{i t+\tau}$ who will be at location $i$ at time $t+\tau$, where $\tau$ is the walking distance time between the user and location.

Since this number $n_{i t+\tau}$ is not the current number of people inside the location, a prediction is needed. $c$ is a 'scale parameter' which sets the effect of $\mathrm{CW}$ on overall location weight $W$.

The above equation is very simple; non-linear equations can also be used. In addition, the average number $n$ of pedestrians at the location for the whole episode duration can be examined, instead of only the instantaneous value of $n$.

3.2.3 User weight: The category types defined in Section 3.1 are generic: one can eat in restaurants, fast food outlets, cafeterias, etc.; shopping can be done in souvenir shops, clothes shops, etc. Defining these generic categories was necessary, in order to reduce the size of the decision network.

However, when a passenger receives a suggestion from the system to perform a discretionary activity, it is also necessary to take into account the fact that these generic categories contain locations which offer very different types of services. Activity types are therefore divided into sub-categories: for example, 'eating' can refer to restaurants, fast food outlets, and bar/ cafeterias. Then a logit model which takes into account several socio-demographic characteristics of passengers (e.g. gender, age, frequent flyer or not, etc.) can be calibrated.
When the system provides passengers with suggestions, it knows to which group those passengers belong, and thus their socio-demographic characteristics. It uses them to obtain the probabilities for each sub-category. The UW for each location is computed by multiplying this probability by scale parameter $u$

$$
\mathrm{UW}_{i}=u P(i) .
$$

Given a certain passenger group, all locations belonging to the same sub-category have the same UW. Unlike base and CWs, this weight does not depend on time $t$.

The logit model parameters should be calibrated with surveys or according to passengers' mobile phone traces.

3.2.4 Airport weight: The airport management may have an interest in advertising some locations more than others, for marketing reasons, to increase revenues, etc. For example, if the management directly controls the smartphone application, which sends suggestions to passengers, it can ask the owners of those commercial activities to pay for publicity in the app.

The airport management should choose a value of advertising factor (af) for every location in the airport - ideally a value between 0 and a fixed maximum number. The app should be designed for easy modification by the airport management. AW is obtained, for every location, by multiplying af by scale parameter $\alpha$

$$
\mathrm{AW}_{i}=\alpha \times \mathrm{af}_{i} .
$$

This weight should not depend on time $t$, but the management might want to assign different values of $a f$, depending on passengers' time horizons and/or time-of-the-day.

Before applying this weight system to a real-world scenario, tests must be carried out to set scale parameters $\mathrm{BW}_{\max }, c, u$ and $\alpha$. These parameters control the importance of every weight in computing overall weight $W$. Further modifications to them can also be made after the smartphone application release, in order to improve the effectiveness of these solutions.

\section{Proof-of-concept case-study}

The case-study presented below is a proof-of-concept, to illustrate how the whole system works.

The six activity types described in Section 3.1 were used in the activity choice model. Time horizon and time unit length were set, respectively, at 180 and $5 \mathrm{~min}$, according to typical arrival curves found in the literature $[2,18,36]$. Passengers were divided into three groups, according to their arrival times:

- Early: arriving between 3 and $2 \mathrm{~h}$ before flight departure time.

- In-time: arriving between 2 and $1 \mathrm{~h} 15 \mathrm{~min}$ before flight departure time.

- Late: arriving between less than $1 \mathrm{~h} 15 \mathrm{~min}$ before flight departure time.

The model cannot be calibrated without real data from passengers' smartphone traces. The utility function parameters for this proof-of-concept case study were set directly by the authors. Parameters were not randomly set; suggestion from the literature $[16,19,29]$ and in some cases, authors' personal experience were applied.

As the logit model could not be used on the universal choice set due to the extremely high number of alternatives, 30 paths were sampled by the authors. Of these, 10 paths were for early arriving passengers, 13 for in-time ones and 7 for late ones.

The logit model containing the utility function parameters was implemented in Matlab. As inputs, the script needs:

- A matrix containing the sampled paths. Each row is a path, with one column for every time unit. In each cell, a number ranging 1 to 6 indicates the activity type.

- Three matrices containing the time-of-the-day, satiation and repetition parameters. 


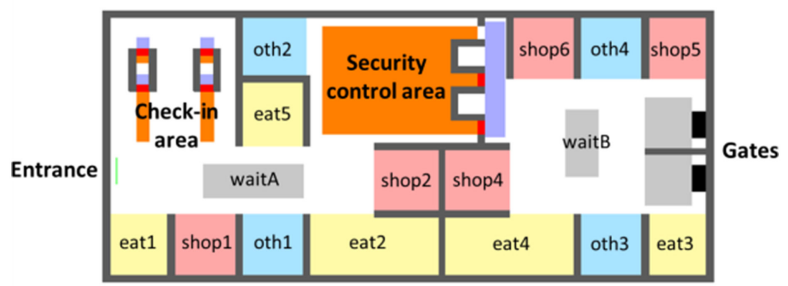

Fig. 3 Proof-of-concept case-study: airport terminal layout

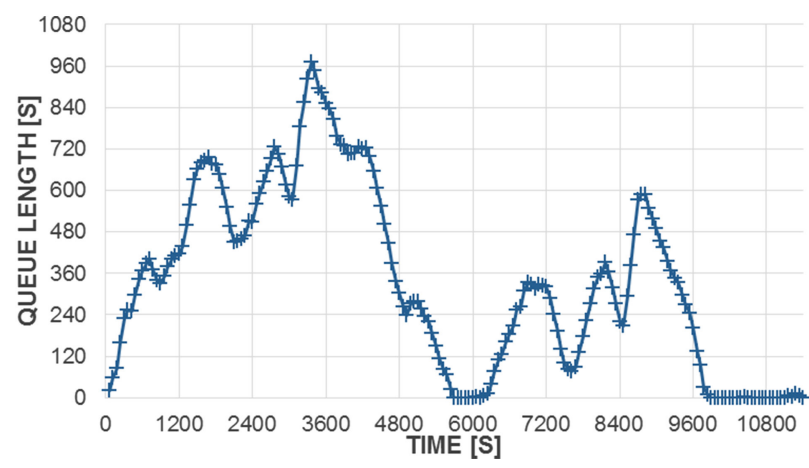

Fig. 4 Prediction of queue length. Average value after five runs

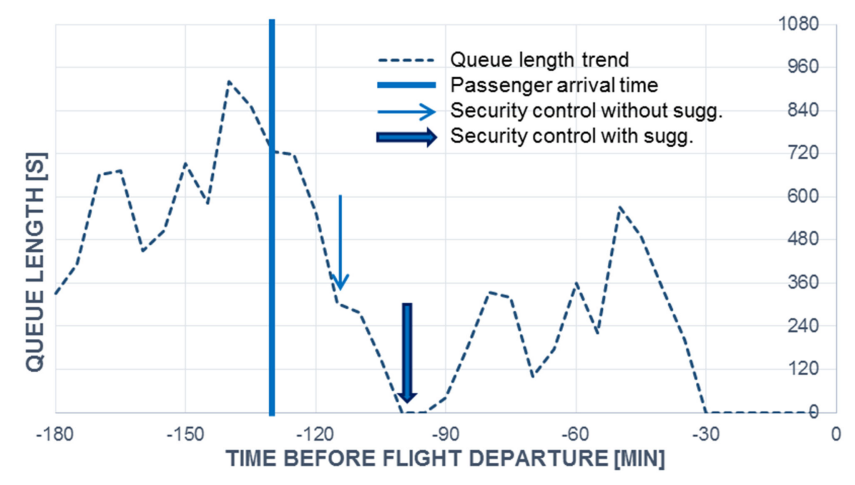

Fig. 5 Suggestion effect on early arriving passenger

The model provides as output the probability of each sequence being chosen by each passenger group.

To represent the movements of each passenger inside the airport terminal, Nomad micro-simulation software, developed at the Delft University of Technology [37-39], was used.

As inputs, this software requires a representation of the airport terminal, the activity sequence probabilities obtained from the activity choice model, and the calibration of a weight system for the location choice.

The airport terminal layout used is shown in Fig. 3. Although it is simplified with respect to a real-world terminal, it is sufficiently complex to avoid this case study to be trivial. In view of the small number of possible destinations for each activity type, location choice was not implemented with the in-built model, but directly by the analyst, in order to have more control over the system.

The simulation consisted of a 15 -min pre-load phase and a 3-h simulation time. 660 pedestrians entered the simulation. The main focus was on 300 of them, representing passengers starting to enter the simulation just after the pre-load phase and boarding a flight at the end of the simulation. The remaining were 300 passengers boarding $1 \mathrm{~h} \mathrm{30^{ \prime }}$ after the pre-load phase and 60 that would have board $4 \mathrm{~h} 30^{\prime}$ after the pre-load phase. Of the 300 passengers of interest, $50 \%$ were in-time arriving passengers, $30 \%$ late passengers and $20 \%$ early passengers. These percentages were chosen according to arrival curves found in the literature [18]. The simulation was run five times. This number was chosen in view of the relatively small variance among the outputs of each run and the relatively long computational time required for each run (about $1 \mathrm{~h}$ 20 using a PC with an Intel ${ }^{\circledR}$ Core ${ }^{\mathrm{TM}} \mathrm{i} 5-6200 \mathrm{U}$ processor running at $2.30 \mathrm{GHz}$, PC RAM was $4.00 \mathrm{~GB}$ ). In a real-world application, the simulation would optimally be run several times, in order to increase prediction precision.

Two outputs were obtained from this simulation: the trend of queue length (Fig. 4) at security control and the occupation trend inside sales areas.

The suggestion generation model was applied to three passengers, one for each type (early, in-time and late arrivals).

The utility function variables had to be modified in order to find the new 'best' activity sequence, substituting expected queuing time (EQT) in the mandatory time-of-the-day part with the predicted queue length trend, obtained from the micro-simulation. The value of dummy variable $I_{\text {info }}$, in the waiting time-of-the-day part of the utility function, was also changed to 1 . To find the sequence with the highest utility for each passenger group, a traditional shortest-path algorithm (Bellman-Ford) was applied, omitting satiation and repetition non-link-additive parameters. The results were still representative, the aim being to give a proof-ofconcept illustration of the whole procedure, although a nonadditive shortest-path algorithm is recommended for a real-world application.

In this way, the system could identify the best sequence for each passenger, as follows. The security control start time is set at a moment when the queue length is short: for example, in Fig. 5 the early passenger is advised to pass through security control $100 \mathrm{~min}$ before flight departure time, whereas without this suggestion he/she would have done it $15 \mathrm{~min}$ earlier, queuing for a longer time. Note that there would have been another no-queue window in the last $30 \mathrm{~min}$ before departure. An activity path containing a mandatory activity that late in the sequence has a much lower modified utility, because the base mandatory time-of-the-day utility is extremely low in the last $30 \mathrm{~min}$ (very few passengers are observed to pass through check-in or security control at that time). In other words, latest times for mandatory activities are addressed implicitly in the model, as no passenger will be observed performing a mandatory activity after a certain time.

The best sequence also contains discretionary activities; in the second level of the suggestion generation model, a specific location was assigned, to each of these activities, with the weight system shown in Section 3.2.

The weight system was implemented in Matlab. The script needs the following inputs:

- suggested activity sequence;

- current time, expressed in minutes until departure time;

- current user position, expressed in distance from all airport locations;

- minimum time $t_{p i}$ to perform an activity, for all locations;

- CWs for every location at every time (given a time discretisation lower than or equal to time unit length);

- AWs for all locations.

Since the passenger groups were only defined according to their arrival time (which is not a socio-demographic characteristic), UWs were not considered. The output was a vector containing all the locations total weights $W$. The location with the highest weight is advised. A location already suggested to a specific user cannot be suggested again.

Lastly, for each of the three passengers, a sequence of activities is obtained, associated (for discretionary activities only) with specific locations. Users receive suggestions so that they can follow this sequence.

To assess the suggestion effect on passengers, Nomad microsimulation software was again used. A new simulation was run; the authors uploaded in the system the three passengers who follow suggestions exactly and three other passengers, generated at exactly the same time, respectively, who follow their original activity sequences, thus ignoring the suggestions. All other pedestrians in the simulation did not receive any kind of information and behaved as they had done in the first runs. After running the simulation five times, a comparison was made between passengers who followed and did not follow the suggestions. The 
Table 1a Passengers' behaviour with and without suggestions. Comparison tables

\begin{tabular}{|c|c|c|c|}
\hline Early arriving passenger & Queuing time & Waiting time & Activity time \\
\hline without sugg. & $44^{\prime \prime} 35^{\prime \prime}$ & $22^{\prime} 01^{\prime \prime}$ & 1 h $27^{\prime} 58^{\prime \prime}$ \\
\hline with sugg. & 0'27" & $01^{\prime} 07^{\prime \prime}$ & 1 h $54^{\prime} 36^{\prime \prime}$ \\
\hline
\end{tabular}

Table 1b

\begin{tabular}{|c|c|c|c|}
\hline In-time arriv. passenger & Queuing time & Waiting time & Activity time \\
\hline without sugg. & $5^{\prime} 52^{\prime \prime}$ & $19^{\prime} 45^{\prime \prime}$ & $51^{\prime} 03^{\prime \prime}$ \\
\hline with sugg & $1^{\prime} 50 "$ & 04'08" & $1 \mathrm{~h} 15^{\prime} 40^{\prime \prime}$ \\
\hline
\end{tabular}

Table 1c

\begin{tabular}{lccc}
\hline Late arriving passenger & Queuing time & Waiting time & Activity time \\
\hline without sugg. & $3^{\prime} 53^{\prime \prime}$ & $2^{\prime} 02^{\prime \prime}$ & $19^{\prime} 49^{\prime \prime}$ \\
with sugg & $4^{\prime} 05^{\prime \prime}$ & $3^{\prime} 03^{\prime \prime}$ & $44^{\prime 2} 27^{\prime \prime}$ \\
\hline
\end{tabular}

aim was to verify the suggestion effect on queuing time at security control, waiting time in front of the gate and activity time (i.e. time spent in other activities).

Table 1 shows these comparisons. Queuing time is reduced for the early passenger who postpones the moment when he/she passes through security control, saving $90 \%$ of the queuing time (see Fig. 5). The in-time passenger also decreases queuing time significantly, whereas the late passenger, in this case, is suggested to go to security control at exactly the same time he/she would have gone anyway, so queuing time remains virtually unchanged. In a real-world application, reducing the queuing time for early passengers would probably be easier than for late ones, because there is more time available in the time horizon to find a good lowqueue window.

Waiting times were also enormously reduced. Within these values, the last $5 \mathrm{~min}$ in the sequence was not considered, because it was assumed that boarding starts $5 \mathrm{~min}$ before departure.

The time saved by reducing queuing and waiting is re-invested by passengers in discretionary activities. The increase in activity time is particularly great, in relative terms, for the late passenger.

This is, of course, an example in which everything was under the analyst's control. In a real airport, without this suggestion system, some passengers move to the gate early because they are worried of losing their flight and they do not know how far they are from their gate; others genuinely want to spend time in front of the gate, (e.g. reading, using the smartphone, contemplating, etc.). With our system, the former passengers will reduce their stress and spend more time in discretionary activities, ideally in the suggested locations. The latter type of passenger at some point will start ignoring discretionary activity suggestions and move to the gate.

This means that the saved queuing time will not be completely re-invested in activity time, but some passengers will actually increase their 'waiting' time. Although that goes against one of the aims of the system (i.e. increasing activity time), it does not just have a negative connotation for airport management, as passengers, improving their airport experience, may be more likely to return to the airport.

We illustrated here the results that this system can achieve and which indicators can show whether the system is working or not.

\section{Conclusion and further research}

This work presents a method to make suggestions to airport passengers, based on user profiles (i.e. passenger groups), in order to reduce queuing and waiting times, thereby improving passengers' satisfaction and increasing airport revenues.

In the system framework (Fig. 1), the behaviour of passengers with different socio-demographic characteristics is modelled with an activity-choice model. Queue lengths and congestion inside commercial areas are predicted thanks to a pedestrian microscopic simulation tool. Passengers are suggested to perform mandatory activities when the predicted queue length is reasonable and specific discretionary activities according to time available, user profiles, location distance, location congestion and airport management preferences. A proof-of-concept case study has been presented in Section 4: passengers' behaviour in both cases of receiving and not receiving suggestion has been simulated. In the first case, passengers experienced less queuing and waiting time, and the time saved was spent in discretionary activities.

Further research will focus on two issues:

- system dynamics;

- users' feedback effect.

The system presented in the case study is static because it deals with a limited user-base and all the predictions made depend on the activity choice model, which is calibrated with historical data, i.e. mobile phone traces. However, app users' traces can be used to regularly re-calibrate the model, adapting it to changes in passengers' behaviours and therefore improving its prediction reliability (red-dashed arrow in Fig. 1). Real-time information on smartphone locations can also be exploited to gather indications on system performance and improve short-term queue length predictions.

Feedback effect is particularly important in choosing the right moment when to suggest mandatory activities. Queue length trend may be significantly different from predictions because passengers modify their behaviour following suggestions. This difference depends, of course, on the number of users receiving and following instructions. Queue length prediction should take into account the behaviour of passengers receiving information (compliance).

The structure of the system presented in this paper is flexible and can be adapted to different pedestrian environments (e.g. railway stations, intermodal centres, malls, exhibitions), to a limited or a wide user-base.

\section{Acknowledgments}

The authors would like to thank Prof. Michel Bierlaire, Riccardo Scarinci and Marija Nikolic from École Polytechnique Fédérale de Lausanne for their support and Mario Carlos Campanella and the Transport \& Planning Department from the Delft University of Technology for the use of Nomad microsimulation tool.

\section{References}

[1] https://www.eurocontrol.int/publications/challenges-growth-2013-reports, accessed November 2017

[2] Guizzi, G., Murino, T., Romano, E.: 'A discrete event simulation to model passenger flow in the airport terminal'. Proc. 11th WSEAS Int. Conf. on Mathematical Methods and Computational Techniques in Electrical Engineering, 2009, pp. 427-434

[3] https://www.icao.int/Meetings/wrdss2011/Documents/JointWorkshop2005/ ATAG_SocialBenefitsAirTransport.pdf, accessed November 2017

[4] http://www.intervistas.com/downloads/reports/Economic $\% 20$ Impact $\% 20 \mathrm{of}$ \%20European\%20Airports\%20-\%20January\%202015.pdf, accessed November 2017

[5] Lin, Y.H., Chen, C.F.: 'Passengers' shopping motivations and commercial activities at airports - the moderating effects of time pressure and impulse buying tendency', Tour. Manag., 2013, 36, pp. 426-434 
[6] Popovic, V., Kraal, B.J., Kirk, P.J.: 'Towards airport passenger experience models'. 7th Int. Conf. on Design \& Emotion, 2010, pp. 1-11

[7] Chung, Y.S., Wu, C.L., Chiang, W.E.: 'Air passengers' shopping motivation and information seeking behaviour', J. Air Transp. Manag., 2013, 27, pp. $25-$ 28

[8] Roanes-Lozano, E., Laita, L.M., Roanes-Macías, E.: 'An accelerated-time simulation of departing passengers' flow in airport terminals', Math. Comput. Simul., 2004, 67, (1-2), pp. 163-172

[9] Schultz, M., Fricke, H.: 'Managing passenger handling at airport terminals individual-based approach for modeling the stochastic passenger behavior'. Proc. 9th USA/Europe Air Traffic Management Research and Development Seminar, ATM 2011, 2011, pp. 438-447

[10] 'Airport of the Future Project (AFP)', available at http:// www.airportsofthefuture.qut.edu.au/, accessed July 2017

[11] 'Proactive Passenger Flow Management for Airports with an Advanced Forecasting System (AERFOR)', available at http://cordis.europa.eu/ project/rcn/194777 en.html, accessed July 2017

[12] 'Door to Door Information for Airports and Airlines (DORA)', available at https://dora-project.eu/, accessed July 2017

[13] Bierlaire, M., Robin, T.: 'Pedestrians choices', in 'Pedestrian behavior' (Harry Timmermans, Bingley, 2009), p. 1

[14] Hoogendoorn, S.P., Bovy, P.H.L. 'Pedestrian route-choice and activity scheduling theory and models', Transp. Res. B, Methodol., 2004, 38, (2), pp. 169-190

[15] Hoogendoorn, S.P., Bovy, P.H.L.: 'Normative pedestrian behaviour theory and modelling'. Transportation and Traffic Theory in the 21st Centrury, 2002, pp. 219-245

[16] Liu, X., Usher, J.M., Strawderman, L.: 'An analysis of activity scheduling behavior of airport travelers', Comput. Ind. Eng., 2014, 74, (1), pp. 208-218

[17] Kalakou, S., Moura, F.: 'Modelling passengers' activity choice in airport terminal before the security checkpoint: the case of Portela airport in Lisbon', Transp. Res. Proc., 2015, 10, pp. 881-890

[18] Ma, W.: 'Agent-based model of passenger flows in airport terminals', $\mathrm{PhD}$ thesis, Queensland University of Technology, 2013

[19] Danalet, A.: 'Activity choice modeling for pedestrian facilities'. $\mathrm{PhD}$ thesis, EPFL, 2015

[20] Ben-akiva, M., Bowman, J.L., Gopinath, D.: 'Travel demand model system for the information era', Transportation, 1996, 23, pp. 241-266

[21] Shiftan, Y.: 'Practical approach to model trip chaining', Transp. Res. Rec., 1998, 1645, (1), pp. 17-23

[22] Bhat, C.R., Singh, S.K.: 'A comprehensive daily activity-travel generation model system for workers', Transp. Res. A, Policy Pract., 2000, 34, (1), pp. $1-22$
[23] Bowman, J.L., Ben-Akiva, M.E.: 'Activity-based disaggregate travel demand model system with activity schedules', Transp. Res. A, Policy Pract., 2000 35, (1), pp. 1-28

[24] Miller, E.J., Roorda, M.J., Carrasco, J.A.: 'A tour-based model of travel mode choice', Transportation, 2005, 32, (4), pp. 399-422

[25] Shiftan, Y.: 'The use of activity-based modeling to analyze the effect of landuse policies on travel behavior', Ann. Reg. Sci., 2008, 42, (1), pp. 79-97

[26] Abou-Zeid, M., Ben-Akiva, M.: 'Well-being and activity-based models', Transportation, 2012, 39, (6), pp. 1189-1207

[27] Shiftan, Y., Ben-Akiva, M.: 'A practical policy-sensitive, activity-based, travel-demand model', Ann. Reg. Sci., 2011, 47, pp. 517-541

[28] Flötteröd, G., Bierlaire, M.: 'Metropolis-Hastings sampling of paths', Transp. Res. B, Methodol., 2013, 48, pp. 53-66

[29] Danalet, A., Bierlaire, M.: 'Importance sampling for activity path choice', 15th Swiss Transport Research Conf. (STRC), Monte Verità, Ascona, Switzerland, April 2015

[30] Ettema, D., Bastin, F., Polak, J., et al: 'Modelling the joint choice of activity timing and duration', Transp. Res. A, Policy Pract., 2007, 41, (9), pp. 827841

[31] Aschenbruck, N., Munjal, A., Camp, T.: 'Trace-based mobility modeling for multi-hop wireless networks', Comput. Commun., 2011, 34, (6), pp. 704-714

[32] Danalet, A., Farooq, B., Bierlaire, M.: 'A Bayesian approach to detect pedestrian destination-sequences from WiFi signatures', Transp. Res. C Emerg. Technol., 2014, 44, pp. 146-170

[33] Hastings, W.K.: 'Monte Carlo sampling methods using Markov chains and their applications', Biometrika, 1970, 57, (1), pp. 97-109

[34] Reinhardt, L.B., Pisinger, D. 'Multi-objective and multi-constrained nonadditive shortest path problems', Comput. Oper. Res., 2011, 38, (3), pp. $605-$ 616

[35] Shahabi, M., Unnikrishnan, A., Boyles, S.D.: 'An algorithm for non-additive shortest path problem'. 93th Annual Meeting of the Transportation Research Board, 2014

[36] Chun, H.W., Mak, R.W.T.: 'Intelligent resource simulation for an airport check-in counter allocation system', IEEE Trans. Syst. Man Cybern. C, Appl. Rev., 1999, 29, (3), pp. 325-335

[37] Hoogendoorn, S., Bovy, P. .L. 'Simulation of pedestrian flows by optimal control and differential games', Optim. Control Appl. Methods, 2003, 24, (3), pp. $153-172$

[38] Campanella, M.C.: 'Microscopic modelling of waliking behaviour', PhD thesis, Technische Universiteit Delft, 2016

[39] Campanella, M.C., Daamen, W., Hoogendoorn, S.: 'User manual of the microscopic pedestrian simulation model Nomad' (Technische Universiteit Delft, Delft, 2015) 